\title{
O-GIcNAcylation enhances anaplastic thyroid carcinoma malignancy
}

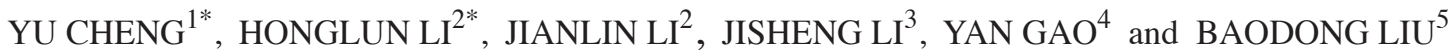 \\ Departments of ${ }^{1}$ Medical Oncology and ${ }^{2}$ Radiology, \\ Affiliated Yantai Yuhuangding Hospital of Qingdao University Medical College, Yantai, Shandong 264000; \\ ${ }^{3}$ Department of Radiology, Penglai Hospital of Traditional Chinese Medicine, Penglai, Shandong 265600; \\ Departments of ${ }^{4}$ Dermatology and ${ }^{5}$ Ultrasound, Yankuang Group General Hospital, Zoucheng, Shandong 273500, P.R. China
}

Received January 15, 2015; Accepted January 26, 2016

DOI: $10.3892 / \mathrm{ol} .2016 .4647$

\begin{abstract}
O-linked $\mathrm{N}$-acetylglucosamine (O-GlcNAc) glycosylation (O-GlcNAcylation), a dynamic post-translational modification of nuclear and cytoplasmic proteins, may have a critical role in the regulation of biological cell processes and human cancer. O-GlcNAcylation is dynamically regulated by O-GlcNAc transferase (OGT) and O-GlcNAc hydrolase (OGA). Accumulating evidence suggests that O-GlcNAcylation is involved in a variety of types of human cancer. However, the exact role of O-GlcNAcylation in tumor pathogenesis or progression remains to be established. Computed tomography scans of patients with anaplastic thyroid carcinoma (ATC) reveal a rapid growth rate and invasion. The present study demonstrated that O-GlcNAcylation accelerates the progression of ATC. The global O-GlcNAc level of intracellular proteins was increased by overexpression of OGT or downregulation of OGA activity with the specific inhibitor Thiamet-G. By contrast, the global O-GlcNAc level was decreased by silencing of OGT. MTT assay indicated that O-GlcNAcylation significantly promotes cell proliferation. Furthermore, O-GlcNAcylation enhanced cellular biological functions, such as colony formation ability, migration and invasion, of ATC cells in vitro. The findings of the present study suggest that $\mathrm{O}-\mathrm{GlcNAcylation}$ is associated with malignant properties of
\end{abstract}

Correspondence to: Miss. Yan Gao, Department of Dermatology, Yankuang Group General Hospital, 560 Mine Road East, Zoucheng, Shandong 273500, P.R. China

E-mail: gaoyan163gaoyan@163.com

Mr. Baodong Liu, Department of Ultrasound, Yankuang Group General Hospital, 560 Mine Road East, Zoucheng, Shandong 273500 , P.R. China

E-mail: liubaodong163@yeah.net

*Contributed equally

Key words: O-linked $N$-acetylglucosamine glycosylation, thyroid carcinoma, O-linked $\mathrm{N}$-acetylglucosamine transferase, proliferation, migration, invasion thyroid cancer, and may be a potential target for the diagnosis and treatment of thyroid cancer.

\section{Introduction}

O-linked $\mathrm{N}$-acetylglucosamine (O-GlcNAc) glycosylation (O-GlcNAcylation) is the modification of serine and threonine groups on nuclear and cytoplasmic proteins by a single residue of O-GlcNAc. Two enzymes are responsible for cyclic O-GlcNAcylation: O-GlcNAc transferase (OGT), which catalyzes the addition of the GlcNAc moiety to target proteins; and $\mathrm{O}-\mathrm{GlcNAc}$ hydrolase (OGA), which removes the sugar moiety from proteins. Analogous to phosphorylation, O-GlcNAcylation changes rapidly and dynamically in response to changes in the cellular environment triggered by extracellular stimuli, such as stress (1), nutrients and cell cycle progression. Furthermore, O-GlcNAcylation is emerging as a key regulator of cellular biological processes, such as transcription, signal transduction, cell motility, morphogens, cell metabolism and development (2-4).

Accumulating studies indicate that perturbations in cellular O-GlcNAcylation levels are involved in a variety of human diseases, such as diabetes and neurological disorders (5-8). In recent years, it has been also determined that O-GlcNAcylation has key roles in the progression and development of certain types of cancer. Caldwell et al observed that O-GlcNAc levels and OGT protein expression increased in the highly metastatic breast cancer cell lines, which suggested that increased O-GlcNAcylation may be closely associated with breast tumor progression (9). Gu et al (10) and $\mathrm{Mi}$ et al (11) demonstrated that the global O-GlcNAc level was markedly elevated in breast, lung and colon tumor tissues compared with matched adjacent tissues; similarly, OGT protein expression correspondingly changed. In addition, clearly enhanced O-GlcNAcylation was observed in the metastatic lymph nodes of breast cancer tissue compared with the corresponding primary tumor tissues $(10,11)$. A growing number of O-GlcNAc-modified proteins with important roles in cell growth, proliferation, motility, differentiation and apoptosis have been identified. Furthermore, numerous oncogene and tumor suppressor gene products, such as c-Fos, c-Jun, c-Myc, retinoblastoma protein and p53, have been revealed 
to be modified by O-GlcNAc (12-14). These results implicate $\mathrm{O}-\mathrm{GlcNAcylation}$ as a crucial regulator that may have an effect on tumorigenesis, migration and metastasis.

At present, thyroid cancer represents $\sim 2.5 \%$ of new cancer diagnoses in the United States and the incidence of malignant thyroid tumor is increasing. Thyroid malignancies are categorized into papillary carcinoma, follicular carcinoma, medullary thyroid carcinoma, anaplastic thyroid carcinoma (ATC), primary thyroid lymphoma (rare) and primary thyroid sarcoma (rare). ATC has the highest mortality rate of all the thyroid tumors, exhibiting a high mitotic rate, and lymphovascular and vascular invasion (15). It rapidly invades surrounding tissues, such as the trachea, or even transfers to the lymph nodes, lungs, liver, chest and femur. Treatment of ATC is typically palliative in its intent, as the disease is rarely curable and almost always fatal, with worse prognoses associated with large tumors, distant metastases, acute obstructive symptoms and leukocytosis. Therefore, ATC management demands rapid, complex and integrated multidisciplinary decision-making, and the underlying malignancy-associated mechanism require further investigation.

Although it has been proposed that aberrant O-GlcNAc metabolism is associated with the malignant properties of a variety of thyroid cancer cell types, and previous studies have indicated that O-GlcNAcylation participates in the functional regulation of ATC cells by modulating Akt1 activity $(16,17)$, the exact roles of O-GlcNAc in thyroid cancer pathogenesis and progression remain to be established.

In the present study, the global O-GlcNAcylation level was altered through OGT overexpression, OGT silencing or OGA inhibition in ATC cells, and the effects of O-GlcNAcylation on the malignant properties of thyroid tumors were determined.

\section{Materials and methods}

Cell cultures and treatments. 8305C ATC cells (European Collection of Authenticated Cell Cultures, Salisbury, UK) were grown in Advanced Minimum Essential medium (MEM; Gibco; Thermo Fisher Scientific, Inc., Waltham, MA, USA) medium supplemented with $2 \mathrm{mM}$ glutamine (Gibco; Thermo Fisher Scientific, Inc.) and 5\% fetal bovine serum (FBS; Gibco; Thermo Fisher Scientific, Inc.) in a humidified atmosphere containing $5 \% \mathrm{CO}_{2}$ at $37^{\circ} \mathrm{C}$. THJ-11T human ATC cells (Cell Resource Center, Institute of Basic Medical Sciences, China Academy of Medical Sciences, Beijing, China) were cultured in RPMI-1640 medium (Gibco; Thermo Fisher Scientific, Inc.) supplemented with $10 \%$ FBS. Both cell lines were treated with $5 \mu \mathrm{M}$ Thiamet-G (Sigma-Aldrich, St. Louis, MO, USA) for $48 \mathrm{~h}$ or the indicated time period.

A vector expressing Flag-tagged human nuclear/cytoplasmic OGT (pCMV-Flag-OGT) was provided by Dr. Jin Won Cho (Department of Biology, Yonsei University, Seoul, South Korea) (18). Transfections were conducted using Lipofectamine 2000 (Invitrogen; Thermo Fisher Scientific, Inc.), according to the manufacturer's instructions.

The shRNA-expressing lentiviral vector pLKO.1-puro, a pCMV $\Delta 8.2$ packaging plasmid construct (encoding gag, pol, rev) and pCMV-VSV-G envelope plasmid were provided by Dr. William C. Hahn (Harvard Medical School and Dana-Farber Cancer Institute, Boston, MA, USA). Small interfering RNA duplexes targeting the coding region of human OGT mRNA sequences were used to suppress OGT enzyme levels in the $8305 \mathrm{C}$ and THJ-11T cells. The human shOGT-targeting sequence was GGATGCTTATATCAA TTTAGG. A control shRNA oligonucleotide sequence that did not match with any known human coding cDNA was used as the control: sense, CCGGTACGTGACACGTTC GGAGAATTCTCGAGAATTCTCCGAACGTGTCACGTT TTTTG and antisense, AATTCAAAAAACGTGACACGT TCGGAGAATTCTCGAGAATTCTCCGAACGTGTCACG TA. shRNA oligos were cloned into the PLKO.1 vector by the AgeI and EcoRI sites. The recombinant plasmid PLKO.1shRNA was cotransfected with $\Delta 8.2$ and VSV-G plasmids into HEK293 cells (Cell Resource Center, Institute of Basic Medical Sciences, China Academy of Medical Sciences), which were used as the virus packaging cells based on their high replication rate and transfection efficiency for efficient virus production. Following $48 \mathrm{~h}$ of transfection, the cell medium was collected and centrifuged at 6,000 x g for $3 \mathrm{~min}$ at $4{ }^{\circ} \mathrm{C}$. The virus supernatant was obtained and used to infect ATC cells. The stable infected cells were selected with $8 \mu \mathrm{g} / \mathrm{ml}$ puromycin (Sigma-Aldrich) for 2 weeks.

Western blotting. Cells were lysed in lysis buffer [50 mM Tris- $\mathrm{HCl}$ (pH 7.4), $150 \mathrm{mM} \mathrm{NaCl}, 1 \%$ NP40, 1 mM EDTA, 1 mM $\mathrm{Na}_{3} \mathrm{VO}_{4}, 10 \mathrm{mM} \mathrm{NaF}$; Sigma-Aldrich] containing a protease inhibitor cocktail (Roche Molecular Diagnostics, Branchburg, NJ, USA) and $5 \mu \mathrm{M}$ PUGNAc (an OGA inhibitor; Toronto Research Chemicals Inc., Toronto, Canada). Proteins were isolated from the total cell lysate by centrifugation at $12,000 \mathrm{x} \mathrm{g}$ for $10 \mathrm{~min}$ at $4^{\circ} \mathrm{C}$, followed by collection of the supernatant. Protein samples $(50 \mu \mathrm{g})$ were separated by $12 \%$ SDS-PAGE (Sigma-Aldrich), transferred to Immobilon-P membranes (EMD Millipore, Billerica, MA, USA), and incubated with antibodies against O-GlcNAc (RL2; mouse monoclonal; dilution, 1:1,000; catalog no., MA1-072; Thermo Fisher Scientific, Inc.), OGT (F-12; mouse monoclonal; dilution, 1:500; catalog no., sc-74546; Santa Cruz Biotechnology, Inc., Dallas, TX, USA) and GAPDH (D-6; mouse monoclonal; dilution, 1:5,000; catalog no., sc-166545; Santa Cruz Biotechnology, Inc.). Following incubation with primary antibodies overnight at $4^{\circ} \mathrm{C}$, the membranes were washed 3 times with phosphate-buffered saline and incubated with horseradish peroxidase-conjugated goat anti-mouse immunoglobulin G secondary antibody (dilution, 1:2,000; catalog no., sc-2005; Santa Cruz Biotechnology, Inc.) overnight at $4^{\circ} \mathrm{C}$. Protein expression was detected using Amersham ECL Prime Western Blotting Detection Reagent (GE Healthcare Life Sciences, Chalfont, UK).

Cell proliferation assay. Cell viability and proliferation were assessed by MTT dye conversion. Briefly, cells were seeded 10,000 cells per well in a 96 -well flat bottom plate. Cells were allowed to grow for $48 \mathrm{~h}$, then $20 \mu \mathrm{l}$ MTT $(5 \mathrm{mg} / \mathrm{ml}$ in phosphate-buffered saline) was then added to each well. After $4 \mathrm{~h}$ incubation at $37^{\circ} \mathrm{C}$, cells were lysed by the addition of $200 \mu \mathrm{l}$ dimethylsulfoxide. Absorbance was measured at $570 \mathrm{~nm}$ using a Tecan Spectra Rainbow Microplate Reader (Tecan Austria GmbH, Grödig, Austria).

Colony formation ability assay. A soft agar colony formation assay was performed as described (2). Briefly, cells $\left(5 \times 10^{3}\right)$ 
A

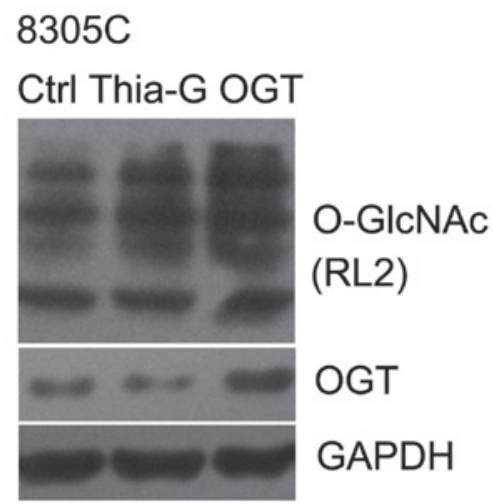

THJ-11T8

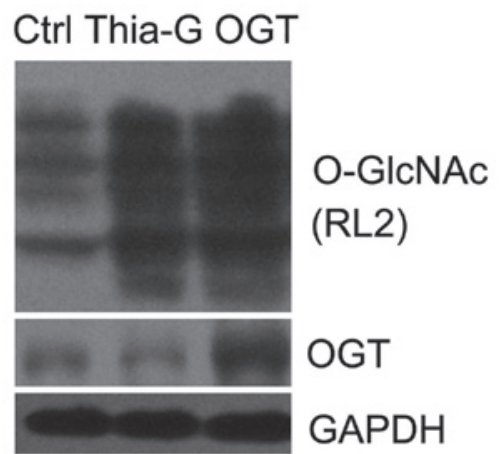

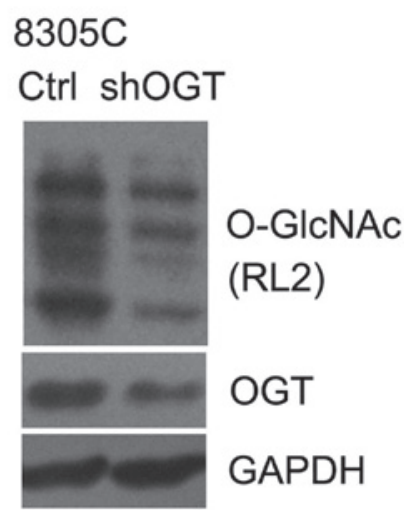

THJ-11T8
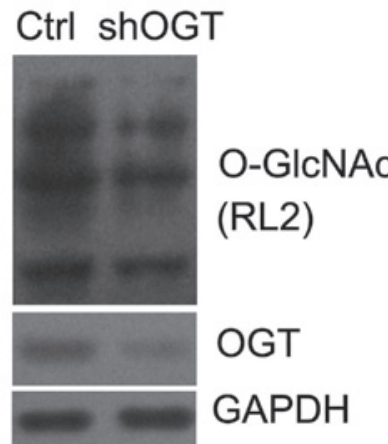

Figure 1. O-GlcNAcylation was elevated by Thia-G treatment or OGT overexpression and decreased by OGT silencing. 8305C and THJ-11T8 cells were pre-treated with (A) $5 \mu \mathrm{M}$ Thia-G for $48 \mathrm{~h}$, pCMV-Flag-OGT or (B) shOGT, and immunoblotted with antibodies against OGT and O-GlcNAc (RL2) to determine the expression of OGT and O-GlcNAcylation. Ctrl, control; Thia-G, Thiamet-G; OGT, O-linked $\mathrm{N}$-acetylglucosamine transferase; O-GlcNAc, O-linked $\mathrm{N}$-acetylglucosamine glycosylation; sh, short hairpin RNA.

were suspended in $1 \mathrm{ml}$ top agar medium (cell culture medium supplied with $0.4 \%$ agar). The cell suspension was then overlaid onto $1.5 \mathrm{ml}$ bottom agar medium (cell culture medium supplied with $0.8 \%$ agar) in six-well tissue culture plates in triplicate. Fresh medium was added to plates every 3 days as a feeder layer. On days $12-18$, the number of colonies was counted in six random fields at X40 magnifications (BX50; Olympus Corp., Tokyo, Japan).

Cell migration assay. A cell migration assay was performed as previously described (19). Cell migration was assayed using Transwell chambers $(6.5 \mathrm{~mm}$; Corning Incorporated, Corning, NY, USA) with $8 \mu \mathrm{m}$ pore membranes. The lower chamber was filled with $600 \mu 1 \mathrm{NIH}-3 \mathrm{~T} 3$ conditioned medium (Cell Resource Center, Institute of Basic Medical Sciences, China Academy of Medical Sciences) with or without OGA inhibitors $\left(5 \mu \mathrm{M}\right.$ Thiamet-G). Cells $\left(5 \times 10^{4}\right)$ were suspended in $100 \mu \mathrm{l}$ medium (MEM or RPMI-1640 with $1 \%$ fetal calf serum) and plated into the upper chamber with or without OGA inhibitors. After $20 \mathrm{~h}$, the number of cells appearing by crystal violet staining (for 20 min; Shanghai Haoran Biological Technology Co., Ltd., Shanghai, China) on the undersurface of the polycarbonate membranes was scored visually in five random fields at x100 magnification using a light microscope (BX50; Olympus Corp.).

Cell invasion assay. For cell invasion assays, the upper face of the membrane was covered with $70 \mu 1$ Matrigel $(1 \mathrm{mg} / \mathrm{ml}$;
BD Biosciences, Franklin Lakes, NJ, USA). The invasion assay was performed using the same procedure as the migration assay, except that the incubation time of the experiment was prolonged to $24 \mathrm{~h}$.

Statistical analysis. All experiments were repeated at least 3 times. Data were analyzed by Student's t-test using SPSS software (version 16.0; SPSS, Inc., Chicago, IL, USA). P<0.05 was considered to indicate a statistically significant difference. Data are presented as the mean \pm standard error of the mean.

\section{Results}

Regulation of O-GlcNAcylation expression in the ATC cell lines. To investigate whether O-GlcNAcylation is important in thyroid cancer malignancy, the level of O-GlcNAcylation was elevated by the overexpression of OGT or the inhibition of OGA, and decreased by the silencing of OGT in the 8305C and THJ-11T ATC cell lines. OGT overexpression (pCMV-Flag-OGT) and Thiamet-G, a highly potent and selective OGA inhibitor, effectively increased the O-GlcNAcylation in $8305 \mathrm{C}$ and THJ-11T cells (Fig. 1A) (20). By contrast, shOGT markedly reduced OGT protein expression levels, as well as O-GlcNAcylation levels, in $8305 \mathrm{C}$ and THJ-11T cells (Fig. 1B).

O-GlcNAcylation increases ATC cell viability. To determine whether O-GlcNAcylation affects cell viability, 8305C and 

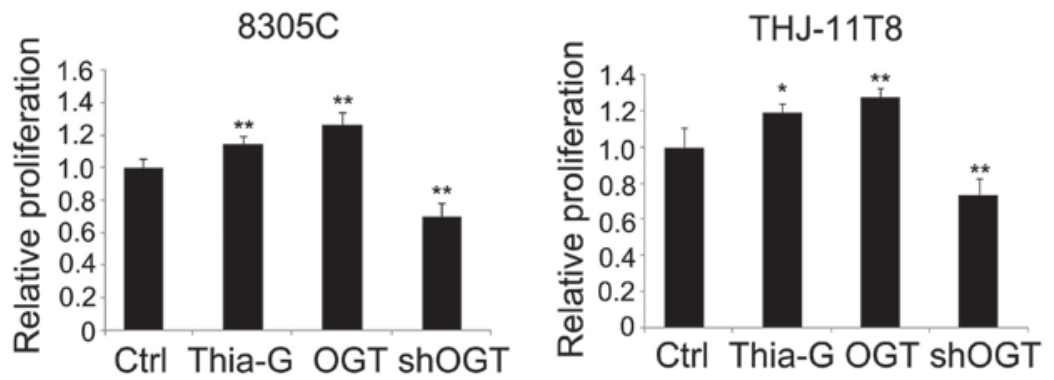

Figure 2. O-linked N-acetylglucosamine glycosylation increases anaplastic thyroid carcinoma cell proliferation. Thia-G and pCMV-Flag-OGT treatment enhanced the cell proliferation of 8305C and THJ-11T8 cells, while OGT silencing with shOGT inhibited cell proliferation. Mean \pm standard error of the mean. ${ }^{*} \mathrm{P}<0.05,{ }^{* *} \mathrm{P}<0.01$ vs. ctrl (Student's t-test). Ctrl, control; Thia-G, Thiamet-G; OGT, O-linked $N$-acetylglucosamine transferase; sh, short hairpin RNA.

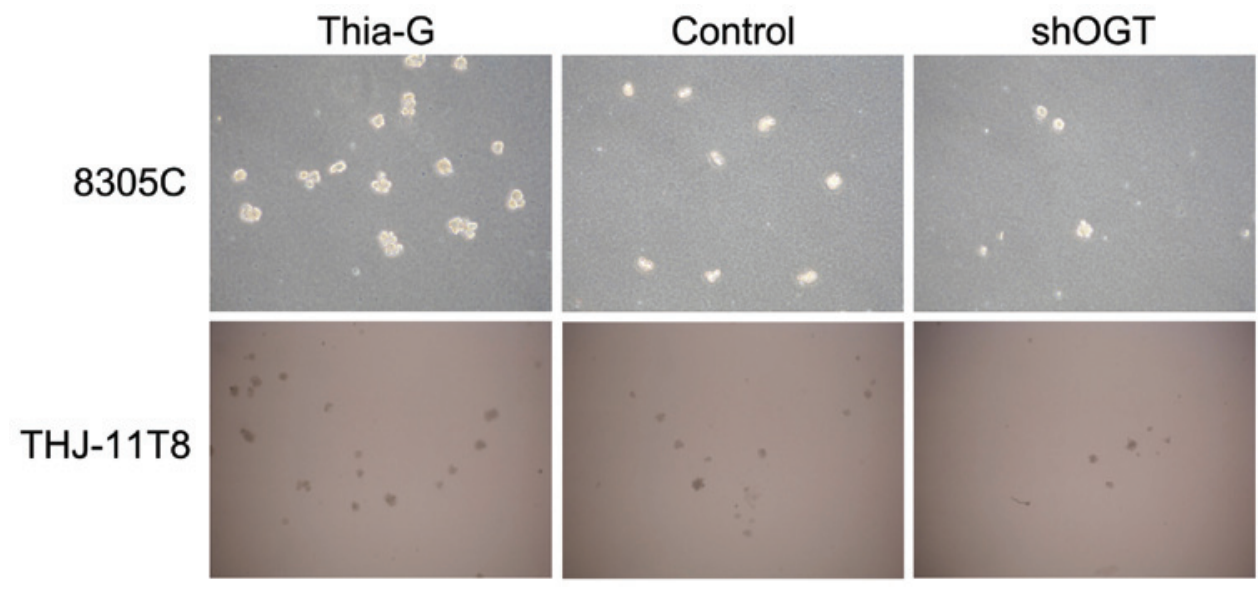

Figure 3. O-linked N-acetylglucosamine glycosylation enhances colony formation ability of anaplastic thyroid carcinoma cells. The anchorage-independent growth of $8305 \mathrm{C}$ and THJ-11T8 cells was analyzed by performing a soft agar colony formation assay. Magnification, $\mathrm{x} 40$. Cells were unstained. Thia-G, Thiamet-G; shOGT, O-linked $N$-acetylglucosamine transferase short hairpin RNA.

THJ-11T ATC cells were treated to produce OGT overexpression (pCMV-Flag-OGT), OGA inhibition ( $5 \mu \mathrm{M}$ Thiamet-G) or OGT silencing (shOGT) cell groups. The cells were grown in medium with serum for $48 \mathrm{~h}$ and an MTT assay for cell proliferation was performed. Proliferation of 8305C and THJ-11T cells increased by $\sim 27$ and $\sim 15 \%(\mathrm{P}<0.01)$ following OGT overexpression, and $\sim 28(\mathrm{P}<0.01)$ and $\sim 19 \%$ $(\mathrm{P}<0.05)$ following OGA inhibition, respectively, relative to the control. Conversely, the proliferation of OGT-silenced $8305 \mathrm{C}$ and THJ-11T cells was decreased by $\sim 30$ and $\sim 27 \%$, respectively, compared with the control $(\mathrm{P}<0.01$; Fig. 2$)$.

O-GlcNAcylation enhances ATC cell colony formation ability. To investigate whether O-GlcNAcylation affects the colony formation ability (anchorage-independent growth) of ATC cells, soft agar colony formations assays were conducted. The assays revealed that OGA inhibition (Thiamet-G) markedly increased the colony formation ability of $8305 \mathrm{C}$ and THJ-11T cells compared with the control. By contrast, OGT silencing (shOGT) markedly reduced the colony formation ability compared with the control (Fig. 3). These results suggest that O-GlcNAcylation is important for maintaining the anchorage-independent growth of ATC cells.

O-GlcNAcylation promotes ATC cells motility. The role of O-GlcNAcylation in thyroid cancer cell metastasis was detected by migration and invasion assays in vitro. The results indicated that OGT overexpression and OGA inhibition significantly enhanced cell migration and invasion in 8305C and THJ-11T cells, whereas OGT silencing significantly inhibited cell migration and invasion compared with the control ( $\mathrm{P}<0.01$; Fig. 4A and B).

\section{Discussion}

The present study analyzed the effect of O-GlcNAcylation in ATC progression and development. The results demonstrated that O-GlcNAcylation enhanced the malignancy of ATC by promoting cellular viability, anchorage-independent growth, migration and invasion, all of which are considered to be among the fundamental properties of malignant cells. These results suggest that the increase of O-GlcNAcylation may initiate and promote ATC formation and metastasis to other lesions.

To change the O-GlcNAc level, three methodological approaches were used. On cells group was transfected with pCMV-Flag-OGT, which efficiently increased OGT protein expression levels, as well as O-GlcNAcylation. The next cells group were treated with Thiamet-G, a potent and selective OGA inhibitor. Prolonged cellular treatment with Thiamet-G is known to elevate O-GlcNAc modification of numerous proteins and has been a useful tool for the investigation of cellular responses affected by O-GlcNAc modification. 
A
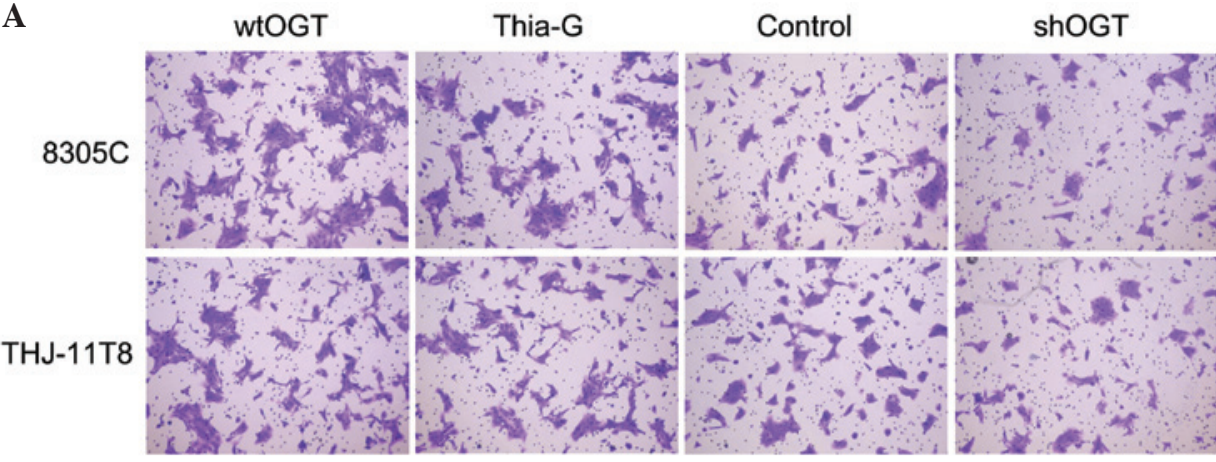

$8305 \mathrm{C}$
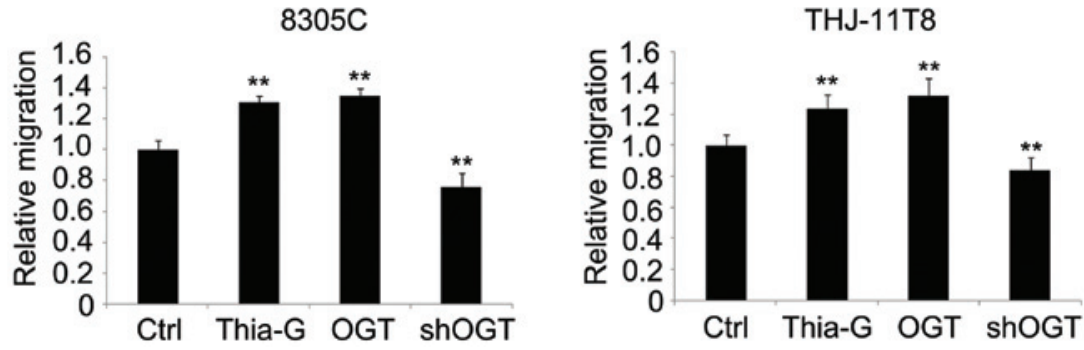

B wtOGT Thia-G Control shOGT
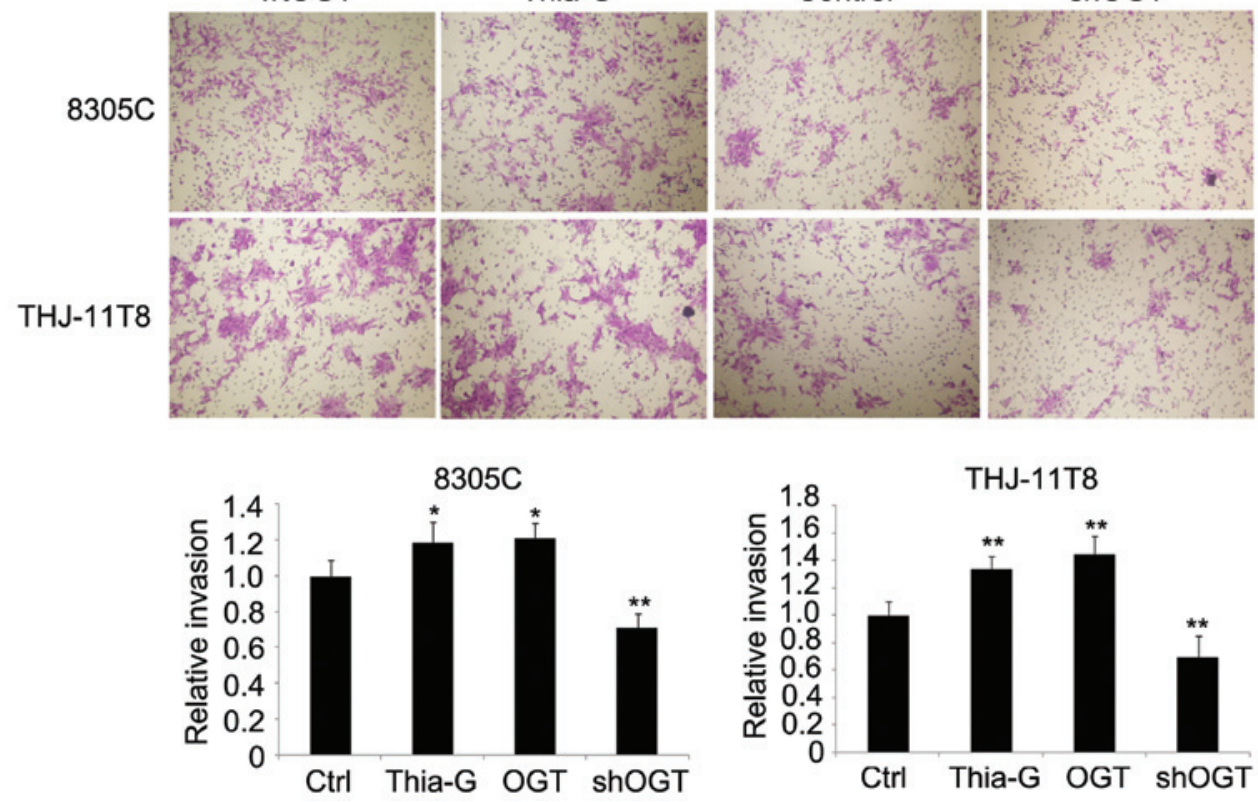

Figure 4. O-linked N-acetylglucosamine glycosylation promotes anaplastic thyroid carcinoma cell motility. OGT overexpression (wtOGT) and Thia-G treatment enhanced the (A) migration (crystal violet staining; magnification, x100) and (B) invasion (crystal violet staining; magnification, $\mathrm{x} 100$ ) of 8305C and THJ-11T8 cells. OGT silencing significantly decreased the migration and invasion of these cells. Mean \pm standard error of the mean. ${ }^{*} \mathrm{P}<0.05,{ }^{* *} \mathrm{P}<0.01$ (Student's t-test). wt, wild-type; Thia-G, Thiamet-G; OGT, O-linked $N$-acetylglucosamine transferase; sh, short hairpin RNA; ctrl, control.

However, Thiamet-G treatment increased O-GlcNAcylation and reduced OGT protein expression, possibly due to the feedback inhibition induced by elevated O-GlcNAcylation. Taking into account the effect of O-GlcNAcylation, the RNA interference method was used to reduce expression of OGT in a third group of cells. All of the methods were similarly effective in altering the O-GlcNAc level in cellular proteins.

At present, the function of O-GlcNAcylation is undergoing thorough investigation. O-GlcNAcylation has important roles in certain human diseases, such as diabetes, neurologic disorders and cardiovascular disease (2). However, there is no unified view as to the role of O-GlcNAc in tumor pathogenesis and progression. Studies by Caldwell et al (9), Gu et al (10) and Mi et al (11) indicated that more O-GlcNAcylation may be closely associated with breast, lung and colon tumor progression. O-GlcNAc levels and OGT protein expression increased in highly metastatic breast cancer cell lines, and lung and colon tumor tissues in comparison with matched adjacent tissues. The lysosomal $\beta$-N-acetylglucosaminidase is a type of O-GlcNAcase. The enhancement of its degradative activity has been observed to be associated with O-GlcNAcylation level in a variety of human cancer types (21-30). Additionally, a previous study proposed OGA activity to be significantly higher and O-GlcNAcylation to be lower in tumor tissue compared with corresponding normal tissue (31). Similarly, a different study demonstrated increased activity of OGA 
activity and decreased O-GlcNAc levels in thyroid cancer compared with benign lesions (16). By contrast, the present study demonstrated that an increase in O-GlcNAcylation was advantageous for the malignancy of ATC cells; however, the two different findings were not mutually contradictory. The former study claimed that O-GlcNAcylation was decreased in tumor tissues, particularly in proteins in the molecular mass range of 45-65 $\mathrm{kDa}$. However, this study also stated that the variety of modified proteins was greater in different molecular mass ranges in the majority of tumor tissues. Therefore, the roles and mechanisms of O-GlcNAcylation in carcinoma development are complex, and may be context-dependent and influenced by cell type or oncogenic events acquired during the course of tumor evolution. O-GlcNAcylation of specific proteins may be more important for the behavior of cancer cells than the global O-GlcNAc level. Thus, further investigations of specific O-GlcNAc modified proteins in tumor cells should be conducted. Simultaneously, to verify the regulatory effect of O-GlcNAcylation on the malignancy of ATC, in vivo studies should also be performed.

O-GlcNAcylation is an inducible and dynamically cycling post-translational modification that can regulate various cellular biological processes $(2,32,33)$; for example, cell cycle, protein stability, apoptosis, growth and the cellular stress response (9,32-34). As a stress sensor, O-GlcNAc levels rapidly and dynamically increase in multiple mammalian cell lines in response to different cellular stresses (35); thus, cells remodel their metabolic and signaling pathways to promote survival. O-GlcNAcylation may protect cells from oxidative stress injury through enhancing Forkhead box $\mathrm{O} 4$ transcriptional activity (36). Raising O-GlcNAcylation may also render cells more thermotolerant (37). In addition, lowering O-GlcNAcylation by reducing OGT levels or blocking hexosamine biosynthetic pathway-sensitized cells to apoptotic stimuli (1). The aberrant metabolism or growth of primary tumor cells is challenged by multiple forms of stimuli and stress, including reactive oxygen species, extracellular matrix components, basement membranes, nutrient deprivation and hypoxia, and attack by the immune system. To defend against microenvironmental death stimuli, tumor cells require metastasis and exposure to novel microenvironments (38). The present study indicates that O-GlcNAcylation elevation may benefit cancer cells to resist apoptotic stimuli, accelerating tumor formation and progression.

In conclusion, the results of the present study demonstrated that O-GlcNAcylation increased the malignancy of anaplastic thyroid carcinoma cells by promoting cellular growth, colony formation, and migration and invasion. Therefore, understanding the association between abnormal O-GlcNAcylation and cancerous behavior may be significant, and O-GlcNAcylation may be a potential target for the diagnosis and therapy of cancer.

\section{References}

1. Zachara NE, O'Donnell N, Cheung WD, Mercer JJ, Marth JD and Hart GW: Dynamic O-GlcNAc modification of nucleocytoplasmic proteins in response to stress. A survival response of mammalian cells. J Biol Chem 279: 30133-30142, 2004.

2. Hart GW, Housley MP and Slawson C: Cycling of O-linked beta- $\mathrm{N}$-acetylglucosamine on nucleocytoplasmic proteins. Nature 446: 1017-1022, 2007.

3. Wells L, Vosseller K and Hart GW: Glycosylation of nucleocytoplasmic proteins: Signal transduction and O-GlcNAc. Science 291: 2376-2378, 2001.
4. Hanover JA: Glycan-dependent signaling: O-linked N-acetylglucosamine. FASEB J 15: 1865-1876, 2001.

5. Dias WB and Hart GW: O-GlcNAc modification in diabetes and Alzheimer's disease. Mol Biosyst 3: 766-772, 2007.

6. Lazarus BD, Love DC and Hanover JA: O-GlcNAc cycling: Implications for neurodegenerative disorders. Int $\mathrm{J}$ Biochem Cell Biol 41: 2134-2146, 2009.

7. Hart GW, Slawson C, Ramirez-Correa G and Lagerlof O: Cross talk between O-GlcNAcylation and phosphorylation: Roles in signaling, transcription, and chronic disease. Annu Rev Biochem 80: 825-858, 2011.

8. Yuzwa SA and Vocadlo DJ: O-GlcNAc and neurodegeneration: Biochemical mechanisms and potential roles in Alzheimer's disease and beyond. Chem Soc Rev 43: 6839-6858, 2014.

9. Caldwell SA, Jackson SR, Shahriari KS, Lynch TP, Sethi G, Walker S, Vosseller K and Reginato MJ: Nutrient sensor $\mathrm{O}-\mathrm{GlcNAc}$ transferase regulates breast cancer tumorigenesis through targeting of the oncogenic transcription factor FoxM1. Oncogene 29: 2831-2842, 2010.

10. Gu Y, Mi W, Ge Y, Liu H, Fan Q, Han C, Yang J, Han F, Lu X and $\mathrm{Yu} \mathrm{W}$ : GlcNAcylation plays an essential role in breast cancer metastasis. Cancer Res 70: 6344-6351, 2010.

11. Mi W, Gu Y, Han C, Liu H, Fan Q, Zhang X, Cong Q and Yu W: $\mathrm{O}-$ GlcNAcylation is a novel regulator of lung and colon cancer malignancy. Biochim Biophys Acta 1812: 514-519, 2011.

12. Zachara NE and Hart GW: Cell signaling, the essential role of O-GlcNAc!. Biochim Biophys Acta 1761: 599-617, 2006.

13. Chou TY, Hart GW and Dang CV: C-Myc is glycosylated at threonine 58: A known phosphorylation site and a mutational hot spot in lymphomas. J Biol Chem 270: 18961-18965, 1995.

14. Kamemura K, Hayes BK, Comer FI and Hart GW: Dynamic interplay between O-glycosylation and O-phosphorylation of nucleocytoplasmic proteins. Alternative glycosylation/phosphorylation of THR-58, a known mutational hot spot of c-Myc in lymphomas, is regulated by mitogens. J Biol Chem 277: 19229-19235, 2002.

15. Hu MI, Vassilopoulou-Sellin R, Lustig R and Lamont JP: Thyroid and parathyroid cancers. In: Pazdur R, Wagman LD, Camphausen KA, Hoskins WJ (Eds): Cancer Management: A Multidisciplinary Approach. 11th edition. UMB Medica, Norwalk, CT, pp1-4, 2008.

16. Krzeslak A, Pomorski L and Lipinska A: Elevation of nucleocytoplasmic beta-N-acetylglucosaminidase (O-GlcNAcase) activity in thyroid cancers. Int J Mol Med 25: 643-648, 2010.

17. Krześlak A, Jóźwiak P and Lipińska A: Down-regulation of $\beta$-N-acetyl-D-glucosaminidase increases Akt1 activity in thyroid anaplastic cancer cells. Oncol Rep 26: 743-749, 2011.

18. Yang WH, Park SY, Nam HW, Kim do H, Kang JG, Kang ES, Kim YS, Lee HC, Kim KS and Cho JW: NF\{kappa\}B activation is associated with its O-GlcNAcylation state under hyperglycemic conditions. Proc Natl Acad Sci USA 105: 17345-17350, 2008.

19. Gu Y, Zhang J, Mi W, Yang J, Han F, Lu X and Yu W: Silencing of GM3 synthase suppresses lung metastasis of murine breast cancer cells. Breast Cancer Res 10: R1, 2008.

20. Yuzwa SA, Shan X, Macauley MS, Clark T, Skorobogatko Y, Vosseller K and Vocadlo DJ: Increasing O-GlcNAc slows neurodegeneration and stabilizes tau against aggregation. Nat Chem Biol 8: 393-399, 2012.

21. Okochi T, Seike H, Higashino K, Hada T, Watanabe S, Yamamura Y, Ito F, Matsuda M, Osafune M, Kotake T and Sonoda T: Alteration of hexosaminidase isoenzymes in human renal carcinoma. Cancer Res 39: 1829-1834, 1979.

22. Narita M, Taniguchi N, Makita A, et al: Elevated activity of beta-hexosaminidase and sulfhydryl modification in the B-variant of human lung cancer. Cancer Res 43: 5037-5042, 1983.

23. Gil-MartinE, Gil-Seijo S,Nieto-Novoa Cand Fernández-Briera A: Elevation of acid glycosidase activities in thyroid and gastric tumors. Int J Biochem Cell Biol 28: 651-657, 1996.

24. Gil-Martín E, Rodríguez-Berrocal FJ, Páez de la Cadena M and Fernández-Briera A: N-acetyl-beta-hexosaminidase activity and isoenzymes in human gastric adenocarcinoma. Oncology 56: 142-154, 1999

25. Luqmani Y, Temmim L, Memon A, Abdulaziz L, Parkar A, Ali M, Baker H, Motawy M and Fayaz S: Measurment of serum $\mathrm{N}$-acetyl beta glucosaminidase activity in patients with breast cancer. Acta Oncol 38: 649-653, 1999.

26. Oktem F, Yazicilar O, Güvenç MG, Toprak M, Uzun H, Aydin S and Uslu E: Urinary N-acetyl-beta-D-glucosaminidase levels in patients with laryngeal squamous cell carcinoma. J Otolaryngol 36: 233-239, 2007. 
27. Wielgat P, Walczuk U, Szajda S, Bień M, Zimnoch L, Mariak Z and Zwierz K: Activity of lysosomal exoglycosidases in human gliomas. J Neurooncol 80: 243-249, 2006.

28. Borzym-Kluczyk M, Olszewska E, Radziejewska I, Lewszuk A and Zwierz K: Isoenzymes of $\mathrm{N}$-acetyl-beta-hexosaminidase in human pleomorphic adenoma and healthy salivary glands: A preliminary study. Clin Chem Lab Med 46: 131-136, 2008.

29. Szajda SD, Snarsk J, Jankowska A, Puchalski Z and Zwierz K: Isoenzymes $\mathrm{A}$ and $\mathrm{B}$ of $\mathrm{N}$-acetyl-beta-D-hexosaminidase in serum and urine of patients with pancreatic cancer. Hepatogastroenterology 55: 695-698, 2008.

30. Olszewska E, Borzym-Kluczyk M, Rzewnicki I, Rutkowska J, Knas M, Rogowski M, Waniewska E and Wielgosz R: Hexosaminidase as a new potential marker for larynx cancer. Clin Biochem 42: 1187-1189, 2009.

31. Slawson C, Pidala J and Potter R: Increased $\mathrm{N}$-acetyl-beta-glucosaminidase activity in primary breast carcinomas corresponds to a decrease in $\mathrm{N}$-acetylglucosamine containing proteins. Biochim Biophys Acta 1537: 147-157, 2001.

32. Hanover JA, Krause MW and Love DC: The hexosaminidase signaling pathway: O-GlcNAc cycling in feast or famine. Biochim Biophys Acta 1800: 80-95, 2010.

33. Butkinaree C, Park K and Hart GW: O-linked beta-Nacetylglucosamine (O-GlcNAc): Extensive crosstalk with phosphorylation to regulate signaling and transcription in response to nutrients and stress. Biochim Biophys Acta 1800: 96-106, 2010.
34. Shafi R, Iyer SP, Ellies LG, O'Donnell N, Marek KW, Chui D, Hart GW and Marth JD: The O-GlcNAc transferase gene resides on the $\mathrm{X}$ chromosome and is essential for embryonic stem cell viability and mouse ontogeny. Proc Natl Acad Sci USA 97: $5735-5739,2000$

35. Dehennaut V, Lefebvre T, Sellier C, Leroy Y, Gross B, Walker S, Cacan R, Michalski JC, Vilain JP and Bodart JF: O-linked $\mathrm{N}$-acetylglucosaminyltransferase inhibition prevents G2/M transition in Xenopus laevis oocytes. J Biol Chem 282: 12527-12536, 2007.

36. Ho SR, Wang K, Whisenhunt TR, Huang P, Zhu X, Kudlow JE and Paterson AJ: O-GlcNAcylation enhances FOXO4 transcriptional regulation in response to stress. FEBS Lett 584: 49-54, 2010.

37. Sohn KC, Lee KY, Park JE and Do SI: OGT functions as a catalytic chaperone under heat stress response: A unique defense role of OGT in hyperthermia. Biochem Biophys Res Commun 322: 1045-1051, 2004.

38. Gupta GP and Massagué J: Cancer metastasis: Building a framework. Cell 127: 679-695, 2006. 